\title{
Hard X Rays from Laser-Wakefield Accelerators in Density Tailored Plasmas
}

\author{
Michaela Kozlova ${ }^{1,2}$ Igor Andriyash ${ }^{1},{ }^{3}$ Julien Gautier, ${ }^{4}$ Stephane Sebban, ${ }^{4}$ Slava Smartsev, ${ }^{3}$ Noemie Jourdain, ${ }^{1}$ \\ Uddhab Chaulagain, ${ }^{1}$ Yasmina Azamoum ${ }^{4},{ }^{4}$ Amar Tafzi, ${ }^{4}$ Jean-Philippe Goddet, ${ }^{4}$ Kosta Oubrerie, \\ Cedric Thaury, ${ }^{4}$ Antoine Rousse ${ }^{4},{ }^{4}$ and Kim Ta Phuoc ${ }^{4, *}$ \\ ${ }^{1}$ Institute of Physics, CAS, ELI Beamlines, Na Slovance 2, 18221 Prague 8, Czech Republic \\ ${ }^{2}$ Institute of Plasma Physics, CAS, \\ Za Slovankou 3, 18221 Prague 8, Czech Republic \\ ${ }^{3}$ Department of Physics of Complex Systems, Weizmann Institute of Science, Rehovot 7610001, Israel \\ ${ }^{4}$ Laboratoire d'Optique Appliquée, ENSTA, CNRS UMR7639, Ecole Polytechnique, \\ Chemin de la Hunière, 91761 Palaiseau, France
}

\begin{abstract}
(Received 13 September 2019; revised manuscript received 8 January 2020;
\end{abstract} accepted 11 February 2020; published 11 March 2020; corrected 30 December 2021)

\begin{abstract}
Betatron x-ray sources from laser-plasma accelerators reproduce the principle of a synchrotron at the millimeter scale. They combine compactness, femtosecond pulse duration, broadband spectrum, and micron source size. However, when produced with terawatt class femtosecond lasers, their energy and flux are not sufficient to compete with synchrotron sources, thus limiting their dissemination and its possible applications. Here we present a simple method to enhance the energy and the flux of betatron sources without increasing the laser energy. The orbits of the relativistic electrons emitting the radiation were controlled using density tailored plasmas so that the energetic efficiency of the betatron source is increased by more than one order of magnitude.
\end{abstract}

DOI: 10.1103/PhysRevX.10.011061

\section{INTRODUCTION}

Betatron x-ray sources, produced from relativistic laserplasma interaction, have the potential to become invaluable tools for modern x-ray applications. Their broadband spectrum, femtosecond duration, and micron source size are ideal features for femtosecond $\mathrm{x}$-ray absorption spectroscopy [1], femtosecond x-ray diffraction [2], or highresolution phase contrast $x$-ray imaging [3-5]. They are also often used as diagnostics for laser-plasma accelerators $[6,7]$. However, they remain marginal in the panel of the commonly used x-ray sources, mainly because of their limited photon energies and relatively low flux. In this paper, we show that the use of density tailored plasmas can dramatically improve the efficiency of betatron sources and push their energy and flux in the typical range of conventional synchrotron facilities.

A betatron source reproduces the principle of a synchrotron in a millimeter-scale laser-produced plasma $[8,9]$. Experimentally, it consists of focusing an intense

\footnotetext{
*Corresponding author. kim.taphuoc@ensta-paris.fr

Published by the American Physical Society under the terms of the Creative Commons Attribution 4.0 International license. Further distribution of this work must maintain attribution to the author(s) and the published article's title, journal citation, and DOI.
}

Subject Areas: Plasma Physics

femtosecond laser into an underdense plasma, usually a low- $Z$ gas jet. As the laser propagates in plasma, its ponderomotive force pushes electrons away from the high-intensity regions and drives an ion cavity in its wake. The cavity, almost free of background electrons, simultaneously acts as an accelerator and a wiggler. Electrons trapped in the cavity are accelerated in the longitudinal direction ( $\hat{z}$, direction of the laser propagation) and are wiggled in the transverse direction $(\hat{x}, \hat{y})$ by strong spacecharge electromagnetic fields. In a typical parameters regime, electrons reach energies of a few hundred $\mathrm{MeV}$ and oscillate with a few hundred micron period and a micron transverse amplitude. When electrons reach relativistic energies, they emit $\mathrm{x}$-ray photons-the so-called betatron radiation. All the features of this radiation depend on the electron orbits, which are defined by the electron Lorentz factor $\gamma(\gg 1)$, its transverse oscillation amplitude $r_{\beta}$, and the background electron plasma density $n_{e}$. The oscillation frequency of the electron is given by $\omega_{\beta}=\omega_{p} / \sqrt{2 \gamma}$, where $\omega_{p}=k_{p} c=\sqrt{4 \pi c^{2} r_{e} n_{e}}$ is a plasma frequency and $r_{e}$ is the classical electron radius [10]. We define the parameter $K=r_{\beta} k_{p} \sqrt{\gamma / 2}$, which is the equivalent of the undulator strength parameter in synchrotrons. For $K \gg 1$, typical for laser-plasma accelerators, the betatron radiation of each electron is emitted into an aperture angle $\theta \approx(1+K) / \gamma$ centered on the laser axis and has a broad synchrotron-type spectrum char- 
acterized by a critical frequency $E_{c}=3 / 2 K \gamma^{2} \hbar \omega_{\beta}$ [11]. The effective number of photons produced by each electron per oscillation period can be estimated as $N_{\mathrm{ph}} \simeq$ $K / 30$ [10].

When produced with tens-of-terawatt class lasers, the betatron radiation is emitted by electrons with energies in the hundred $\mathrm{MeV}$ range, undergoing a few oscillations with $K \sim 10$. The x-ray pulse duration is of the order of the duration of the electron bunch $[2,12]$. The source delivers few femtosecond $x$-ray pulses with a broadband spectrum extending up to a few $\mathrm{keV}$ and containing about $10^{5}$ photons/shot/0.1\% Band width (BW) at $1 \mathrm{keV}$ [13-16]. From the discussion above follows that to further increase $E_{c}$ and $N_{\mathrm{ph}}$ it is necessary to increase $\gamma, r_{\beta}, \omega_{\beta}$, or the electron bunch charge. The most straightforward way is to raise the laser power. It results in an increase of $\gamma$, the bunch charge, and the propagation length [17-19]. The betatron emission becomes therefore brighter, more energetic, and more collimated [20-23]. This is the best method to increase the peak brightness of the source, but it comes at the cost of a lower repetition rate, presently inherent to the large-scale lasers, which is unattractive for applications. A promising alternative to raising the laser power is to modify the electrons' orbits. Electrons' orbits in laserplasma betatron sources are mainly governed by the plasma fields [24] and can therefore be modified by tailoring the plasma density profile along the laser propagation axis. The possibility to enhance the betatron source by plasma density tailoring was discussed theoretically in Ref. [25]. It was shown that the values of $\gamma, r_{\beta}$, or $\omega_{\beta}$ can be significantly increased for an appropriate choice of the density profile. For example, a recent study has shown that the refraction of the laser on a tilted density shock can result in an increase of $r_{\beta}$ and a significant $\mathrm{x}$-ray signal enhancement [26]. Here we present the experimental demonstration of a simple and efficient density tailoring technique to simultaneously increase $\gamma, r_{\beta}$, and $\omega_{\beta}$. We show that the photon energy and the total radiated energy of the betatron source can both be increased by an order of magnitude with respect to the commonly used constant density plasma, further referred to as the reference case.

\section{RADIATION ENHANCEMENT WITH TAILORED DENSITY PROFILES}

Figure 1 schematically shows how density tailored plasmas can be used to enhance $\gamma, r_{\beta}$, and $\omega_{\beta}$, and consequently increase $E_{c}$ and $N_{\mathrm{ph}}$ according to the expressions given above. In Fig. 1(a) the plasma density profile is a longitudinal density up ramp along the laser propagation axis ( $\hat{z}$ axis). Here, as the laser propagates through the plasma, the cavity radius decreases as $R_{c} \propto n_{e}^{-1 / 2}$ and the plasma fields increase as $E_{p} \propto \sqrt{n_{e}}$. The advantage of the up ramp is that it counteracts the electron-wake dephasing which occurs when electrons, traveling faster than the laser in the plasma,
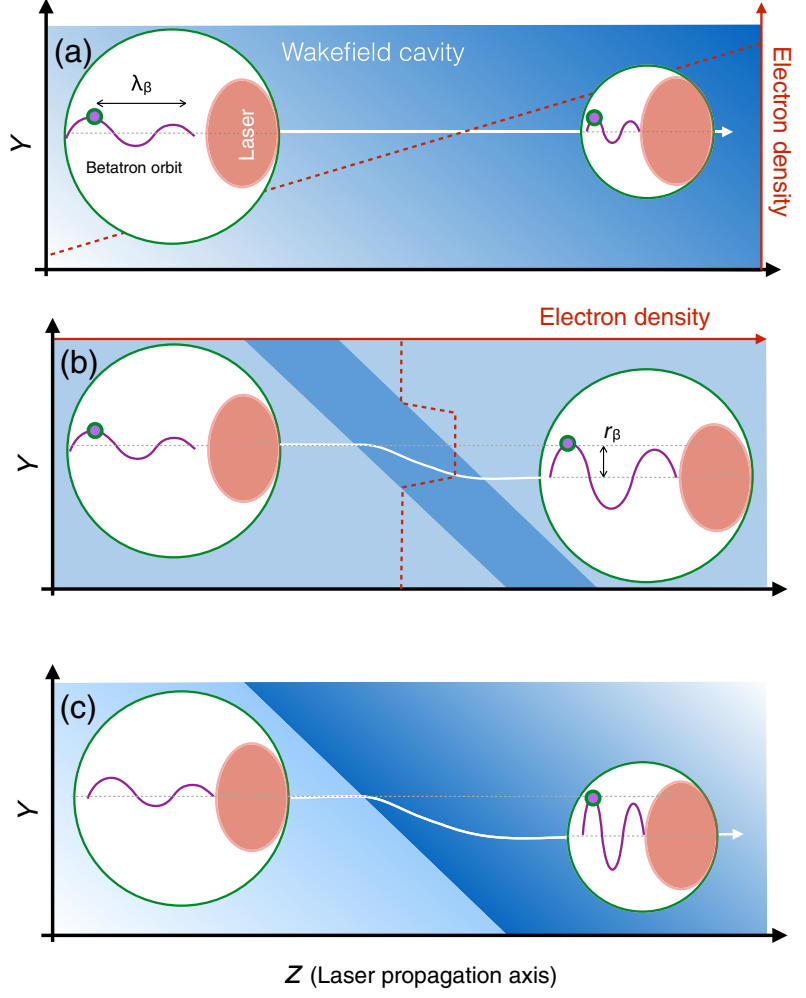

FIG. 1. Schematic representation of the role of density gradients to improve the efficiency of the betatron source. (a) A longitudinal density gradient results in an increase of $\gamma$ due to rephasing and an increase of the betatron oscillation frequency $\omega_{\beta}$. (b) A sharp transverse density gradient results in a shift of the cavity axis due to the refraction of the laser pulse and in an increase of the oscillation amplitude $r_{\beta}$. (c) Combining longitudinal and transverse density gradients increases simultaneously $\gamma, \omega_{\beta}$, and $r_{\beta}$.

overrun the plasma wake (middle of the ion cavity) and start deccelerating. The density up ramp allows us to maintain electrons in the strongest field at the back of the cavity over a longer distance, therefore increasing $\gamma$ with respect to the reference case [27,28]. It is important to mention that the simultaneous increase of the plasma density and the electron energy can be balanced to preserve the oscillation frequency $\omega_{\beta} \propto \sqrt{n_{e} / \gamma}$, or even increase it (if $n_{e}$ grows faster than $\gamma$ ). In this case, along with an increase of $\omega_{\beta}$, one may also anticipate increase of $K$ through $\gamma$.

Figure 1(b) shows how a density gradient along the transverse (e.g., along $\hat{y}$ ) direction provides another degree of optimization. When the laser encounters a tilted density slab it is refracted in one direction at the front interface and in the other direction at the back interface of the slab. As a result, the laser axis and the ion cavity are both shifted transversely [26]. The amplitude of the shift depends on the density, the thickness, and the angle of the slab with respect to the laser axis. When the density gradient is sufficiently sharp, $l_{\text {grad }} \lesssim \lambda_{\beta}=2 \pi c / \omega_{\beta}$, the $\hat{y}$ positions of the laser and 
the ion cavity change abruptly. In that case, the relativistic electrons experience a significant sidekick and their oscillation amplitude is increased. A sharp transverse gradient will therefore result in an increase of $K$ through $r_{\beta}$.

In Fig. 1(c) longitudinal and transverse density gradients are combined so that $\gamma, r_{\beta}$, and $\omega_{\beta}$ are simultaneously increased. This is the most favorable configuration to maximize the efficiency of the betatron source.

For all cases [Figs. 1(a)-1(c)], the modification of the electrons' orbits by the density gradients also results in changes of the spatial profile of the x-ray beam. If we consider the values of $\gamma_{(1,2)}, n_{e(1,2)}$, and $\vec{r}_{\beta(1,2)}$, denoted 1 and 2 , respectively, corresponding to the cases without and with density tailoring, the beam divergence $\theta$ will be modified such that $\left(\theta_{2} / \theta_{1}\right)=\sqrt{\left(n_{2} / n_{1}\right)} \sqrt{\left(\gamma_{1} / \gamma_{2}\right)} \times$ $\left(\vec{r}_{\beta_{2}} / \vec{r}_{\beta_{1}}\right)$. Together with the spectra measurements, the changes of the beam divergence are therefore signatures of the modification of the electrons' orbits due to the density gradients.

\section{RESULTS}

The experiment has been performed at Laboratoire d'Optique Appliquée using a $50 \mathrm{TW} / 30 \mathrm{fs}$, linearly polarized (along $\hat{x}$ axis) laser focused with a $f / 20$ parabolic mirror onto a gas target containing a mixture of helium $(99 \%)$ and nitrogen (1\%) gases. The mixture is used for ionization injection whose advantages for the production of betatron radiation are discussed in Ref. [16]. Furthermore, it is the optimal injection mechanism in the case of an upramp density profile, where commonly considered mechanisms (down ramp or shock injection, self-injection) are inefficient. Accelerated electrons were bent toward a scintillator screen using the static magnet $(1 \mathrm{~T}$ field over $40 \mathrm{~cm})$. However, in our experiment $\left(n_{e}>10^{19} \mathrm{~cm}^{-3}\right.$, gas length $\sim 5 \mathrm{~mm}$ ) the goal was to maximize the production of betatron radiation, and the propagation length exceeds the dephasing length. The measured spectra were therefore not representative of the energy of the electrons when they emit most of the betatron radiation. $\mathrm{X}$ rays were observed using either a deep-depletion $\mathrm{x}$-ray $\mathrm{CCD}$ (for radiation up to $15 \mathrm{keV}$ ) or a scintillator screen imaged with a 16-bit camera (for radiation up to $100 \mathrm{keV}$ ). Measurements of the x-ray flux through metallic filters was used to characterize the betatron radiation in the range $1-10 \mathrm{keV}$. Pairs of Ross filters were used in the range from 10 to $70 \mathrm{keV}$. Plasma density profiles were estimated using a Nomarsky interferometer. A second laser pulse $(300 \mathrm{~mJ} / 30 \mathrm{fs})$ was used as a machining beam to estimate where electrons are injected and where $\mathrm{x}$-ray radiation is produced along the laser propagation axis $[29,30]$.

\section{A. Slow longitudinal density ramp}

We first studied the case of a slow longitudinal gradient. For this, we compared the betatron radiation from two
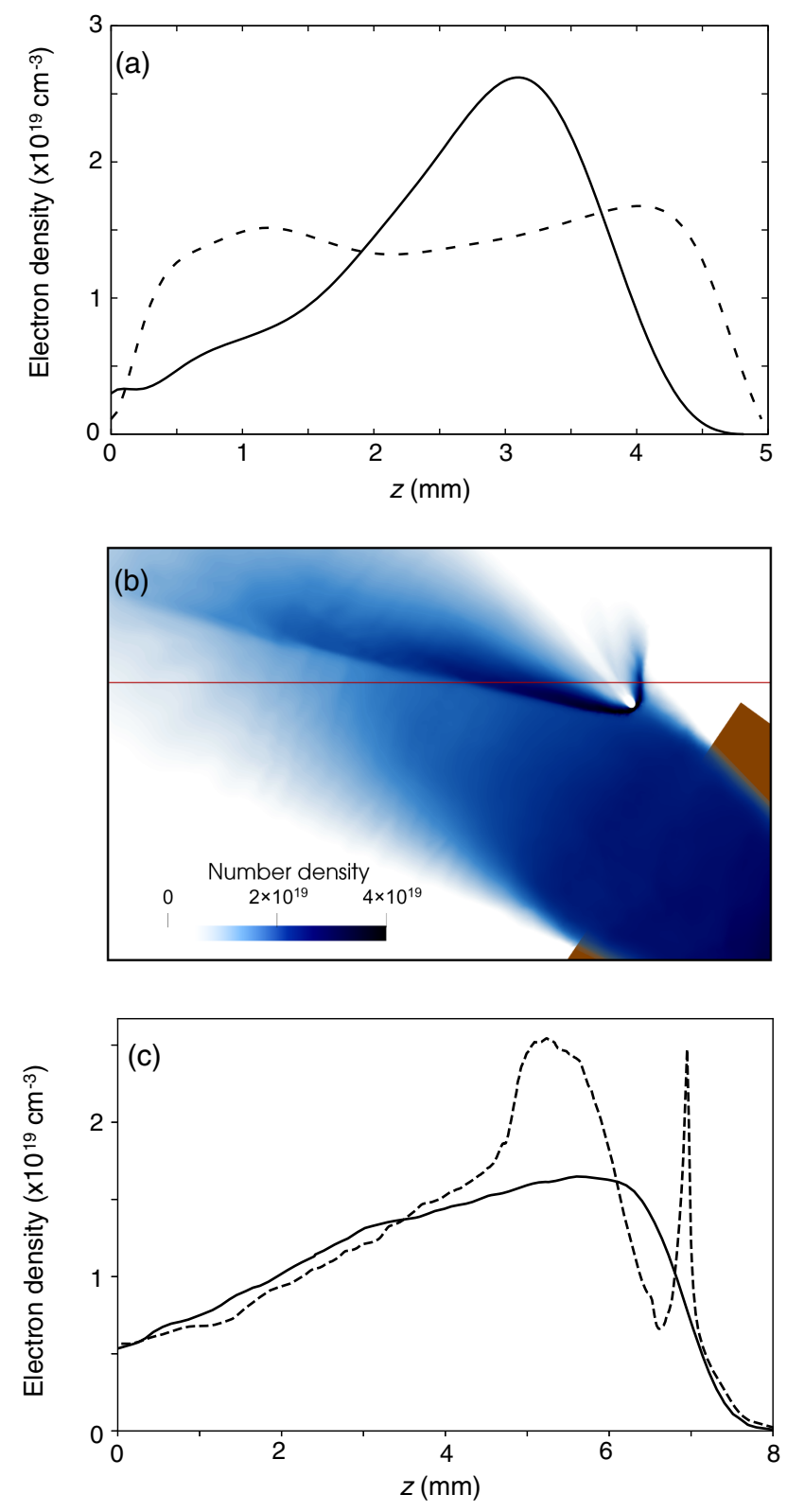

FIG. 2. Measurements and simulations of the density profiles used to improve the efficiency of the betatron source. (a) Measured density profiles of the gas used for the reference case (dotted line) and the longitudinal gradient (solid line). The beveled and tilted gas jets were used to produce the longitudinal gradient. (b),(c) OpenFoam simulation of the transverse gradient obtained by placing a $100 \mu \mathrm{m}$ wire on top of the nozzle. Density profile along the laser propagation (red line) without (solid line) and with (dotted line) the wire.

nozzles: one with a constant density profile and the other with an up-ramp density profile. The density measurements are shown in Fig. 2(a). In both cases the x-ray emission was maximized by adjusting the nozzle position with respect to the laser focus and the gas pressure. We observed an increase of the flux by a factor of $\simeq 3$ without noticeable changes in the $\mathrm{x}$-ray beam profile. In order identify the 


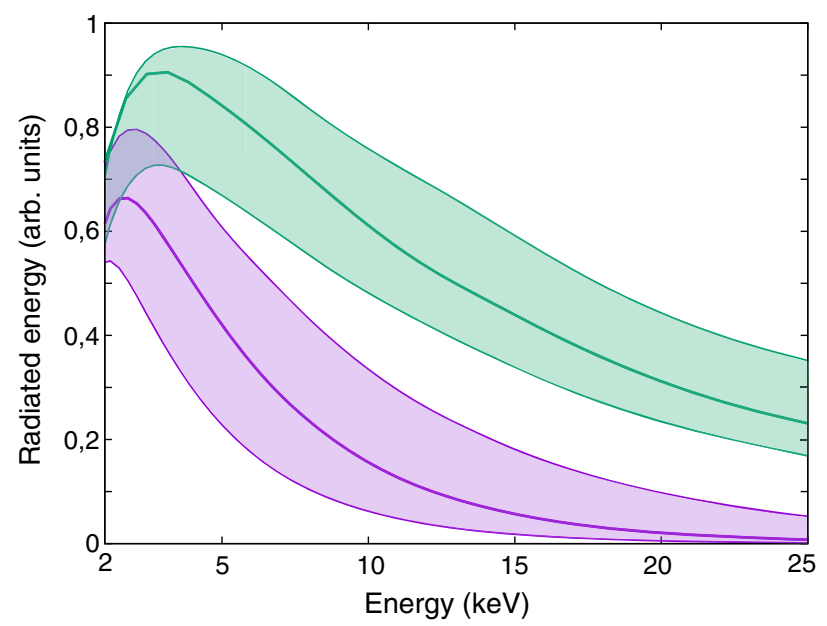

FIG. 3. Measurements of the betatron radiation spectra for a constant density plasma (purple) and for an up-ramp density plasma (green). The shaded area represents the intervals of betatron spectra that fit the $\mathrm{x}$-ray signal measured through metallic filters. The thick solid lines within these areas are the fits obtained from particle-test simulations. Only the density gradient, set as initial condition, distinguishes the two cases in the numerical simulation. The experimental measurements and numerical fit agree and demonstrate that a longitudinal density gradient enhances the energy and flux of the betatron radiation. The critical energy $E_{c}$ characterizing the spectrum shifts from 5 to $10 \mathrm{keV}$.

origin of the flux enhancement, it was measured through an array of aluminium, copper, and titanium filters. The range of betatron spectra that can fit the measurements is represented by the shaded areas in Fig. 3. As expected, the critical energy of the betatron radiation is increased. It shifts from $\simeq 5$ to $\simeq 10 \mathrm{keV}$. In order to make sure that the flux enhancement results from the change of the electron orbits associated with the up-ramp density, we estimated the x-ray emission regions using the method described in Ref. [29]. We found that the plasma lengths over which betatron radiation is produced were $1.5 \pm 0.5 \mathrm{~mm}$ for the up-ramp density and $2 \pm 0.5 \mathrm{~mm}$ for the constant density cases. Such difference of the propagation lengths cannot account for the observed flux enhancement. This confirms the role played by the density gradient.

This result is supported by test-particle simulations based on the ideal ion cavity model [31]. With this simplified approach we have first identified the betatron spectrum that fits into the spectral interval of Fig. 3 for the constant density case. It was obtained using as initial conditions $r_{\beta}=1.25 \mu \mathrm{m}, n_{e}=10^{19} \mathrm{~cm}^{-3}$, and a $2 \mathrm{~mm}$ propagation distance (this choice of parameters is not exclusive, but this has no importance since we focus on the relative differences in the spectra calculated with and without gradient). The spectrum for the up-ramp is well reproduced using the same initial conditions, but with the density profile that increases from $10^{19} \mathrm{~cm}^{-3}$ to $2 \times 10^{19} \mathrm{~cm}^{-3}$ over $2 \mathrm{~mm}$.

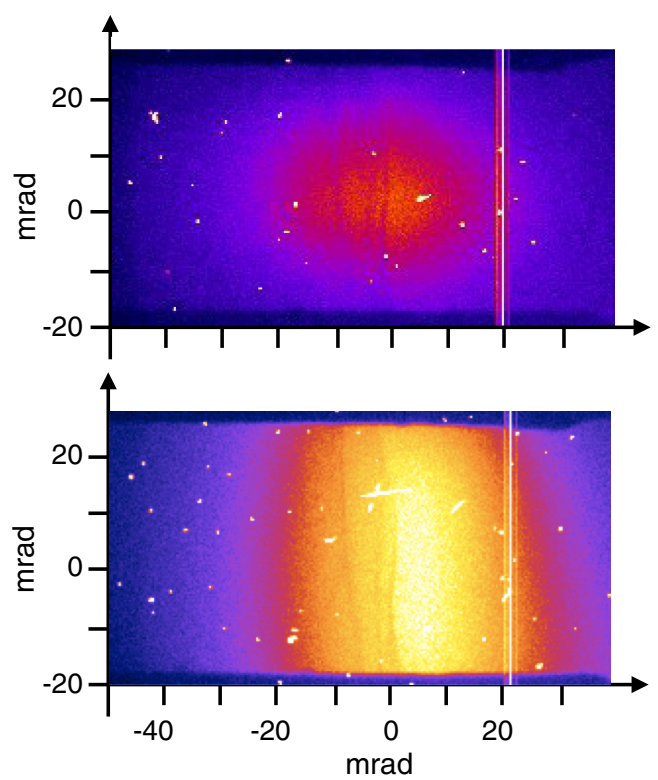

FIG. 4. Measurements of the x-ray beam profiles corresponding to the plasma density profiles displayed in Fig. 2(c). The upper image corresponds to the case without the wire and the lower image to the case with the wire. The images were obtained by imaging a scintillator screen intercepting the x-ray beam (the vertical line is a damage on the camera). The fact that the beam divergence is increased in one direction only demonstrates that the energy $\gamma$ and the transverse amplitude of oscillation $r_{\beta}$ of the electrons are both increased thanks to the density gradient produced by the wire.

\section{B. Sharp tilted density ramp}

The effect of the sharp tilted density gradient was then studied. It was created by inserting a $100 \mu$ m diameter wire into the gas flow of a tilted supersonic nozzle. The density profile obtained reproduces the case of Fig. 1(c). For an accurate estimate of the density profile, we performed twodimensional gas flow modeling using the OpenFoam software [32]. The result is shown in Figs. 2(b) and 2(c).

We first measured the $\mathrm{x}$-ray beam profile with and without the wire. Figure 4 presents typical results. Without the wire, the $\mathrm{x}$-ray beam is quasicircular with a mean FWHM divergence $\theta_{r} \simeq 42 \pm 2 \mathrm{mrad}$. When the wire is inserted, the radiation profile becomes elliptical with a main axis along the $\hat{y}$ direction. The mean divergences are $\theta_{x}=38 \pm 2 \mathrm{mrad}$ and $\theta_{y}=84 \pm 3 \mathrm{mrad}$.

The essential features of the electrons' orbits can be inferred from a simple analysis of the x-ray beam profile [33]. The divergence scales as $r_{\beta} \sqrt{n_{e} / \gamma}$. Since it remains almost constant along the $\hat{x}$ axis while the density is increased, it implies that $\gamma$ is increased. We could estimate an electron energy gain of the order of $50 \%-100 \%$. In the direction of the density gradient tilt, the radiation divergence is significantly increased. This is the signature of an increase of the electron oscillation amplitude $r_{\beta_{y}}$ along the $\hat{y}$ axis. The measured asymmetry translates into the ratio of 


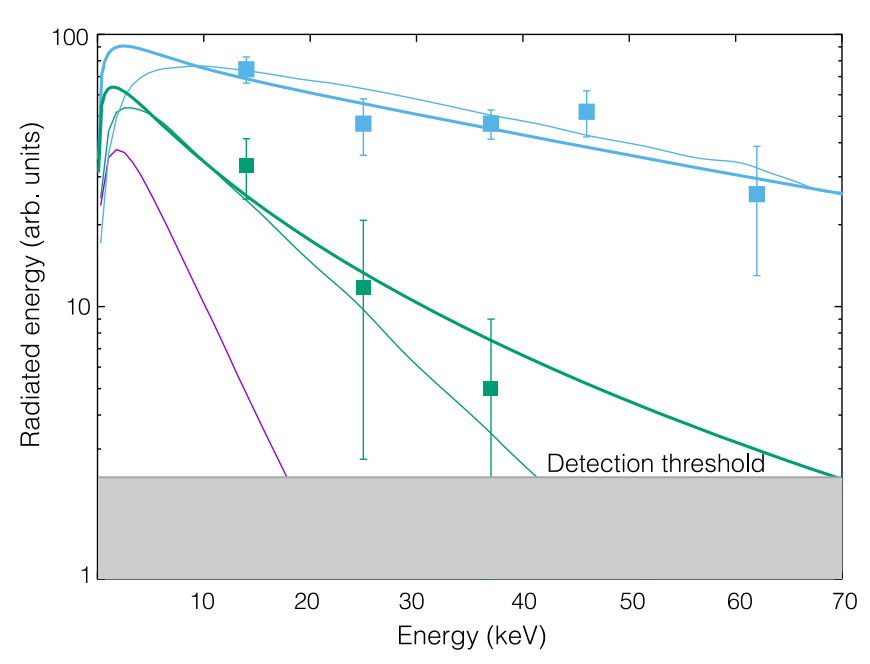

FIG. 5. Betatron radiation spectra measured for the constant density (purple), longitudinal gradient (green), and combined longitudinal and transverse gradients (blue). Thin and thick lines respectively correspond to the test-particle and particle-in-cell simulations. As expected, the increase of $\gamma$ and $r_{\beta}$ deduced from Fig. 4 results in a shift of the radiation spectrum toward higher energies. The critical energies shift from 5 to 10 and to $50 \mathrm{keV}$ for, respectively, for the purple, green, and blue fits.

the oscillation amplitudes $r_{\beta_{y}} / r_{\beta_{x}}=\theta_{y} / \theta_{x} \simeq 2.2$. This analysis confirms that the transverse density gradient allows us to increase both $\gamma$ and $r_{\beta}$.

The spectra with and without wire were measured using Ross filter pairs. They are presented in Fig. 5 together with the reference spectrum. The thin solid lines correspond to the spectra obtained using the test-particle simulations with the same parameters as before, but with $r_{\beta}=2.5$ in the case of the wire, which is in good agreement with the ratio deduced from the $\mathrm{x}$-ray beam profiles. We can estimate the critical energies at 50 and $10 \mathrm{keV}$ with and without the wire, respectively, and at $5 \mathrm{keV}$ for the reference case. The total radiated $\mathrm{x}$-ray energy is further increased by a factor of $\sim 4$ when using the wire. Although a parametric study of the gradient features was not performed (because of the complex interplay between the different parameters such as the gradient length, sharpness, and angle), we found that the effect was sensitive to the wire $\hat{z}$ position within a $\pm 500 \mu \mathrm{m}$ interval, the ascending density gradient must be sharp to the level of typically 100 microns, and the wire position defines the energy and the charge of the electron bunch when it encounters the transverse density gradient.

\section{Particle-in-cell simulations}

For additional insight into the physics of $\mathrm{x}$-ray enhancement, we performed particle-in-cell simulations. We used the quasi-3D pseudospectral code FBPIC [34]. The target was considered to be a fully ionized $\mathrm{He}$ plasma with $2 \%$ of $\mathrm{N}^{+5}$ ion plasma. The density profile is an asymmetric Gaussian density profile defined by $n(z<0)=n_{0} \exp \left(-z^{2} / L_{l}\right)$,
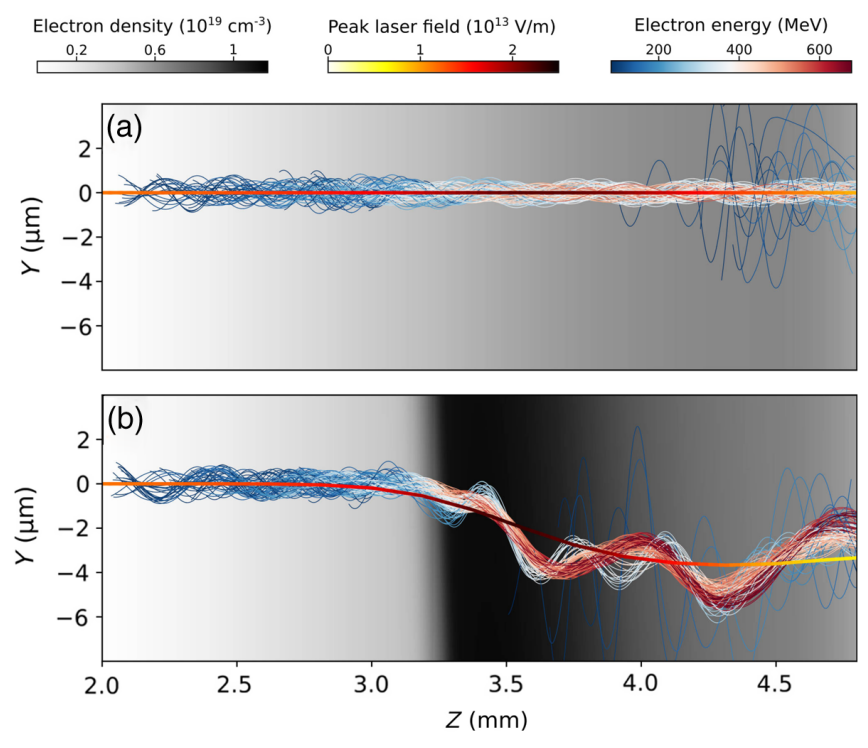

FIG. 6. Particle-in-cell simulations showing the laser propagation (thick curve) and the particle trajectories (thin curves) in $(z, y)$ plane. The orbits are colored according to the laser peak field and the particle energy, respectively. Gray levels represent plasma density. (a) Up-ramp gradient. (b) Up-ramp gradient and transverse density gradient.

and $n(z>0)=n_{0} \exp \left(-z^{2} / L_{r}\right)$, where $L_{l}=3 \mathrm{~mm}$ and $L_{r}=1 \mathrm{~mm}$. The peak density is $n_{0}=1.64 \times 10^{19} \mathrm{~cm}^{-3}$. We considered the cases of gas flow without and with the tilted shock (gray colors in Fig. 6). The shock with a peak density $n_{0} / 2$ is added at $z_{s}=-1.2 \mathrm{~mm}$; it has an asymmetric Gaussian density profile with $L_{\mathrm{sl}}=0.1 \mathrm{~mm}, L_{\mathrm{sr}}=0.7 \mathrm{~mm}$. The angle between shock and laser estimated from gas flow simulations is $\phi_{s}=20$, and this tilt is introduced by replacing $z \rightarrow z-z_{s}+y \tan \phi_{s}$.

Figure 6 shows the plasma density in gray scale in the $(z, y)$ plane, as well as the laser centroid (thick curve) and particles (thin curves) trajectories, colored according to the laser peak field and the particle energy, respectively. When only the longitudinal gradient is considered [see Fig. 5(a)], the acceleration is continuous and the oscillations' amplitudes do not change significantly during the interaction. When the transverse gradient is added in Fig. 5(b), the tilt produces laser refraction and leads to the displacement of the propagation axis by $\approx 4.5 \mu \mathrm{m}$. It induces a kick onto accelerated electrons increasing their amplitude of oscillation $r_{\beta}$. Moreover, the sharp rise of the plasma density relocates particles to higher accelerating and focusing fields, which boosts electrons' energies and induces higherfrequency oscillations.

The spectra for each case were calculated to estimate the overall effect on the betatron emission. They are shown with the thick solid curves in Fig. 5. A good agreement with the experimental measurements in the photon energy distributions (blue and green curves) is obtained. The total radiation energy produced per charge is increased by a 
factor of $\sim 3$, which is close to the experimental values. The estimates of the critical photon energies $\hbar \omega_{c}$, deduced by fitting the calculated spectra, are 14 and $46 \mathrm{keV}$ for the cases without and with the tilted shock, respectively. For these cases the normalized angle-integrated source brightness at $30 \mathrm{keV}$ is $3.4 \times 10^{-5}$ and $2 \times 10^{-4}$ photons per $0.1 \%$ BW per electron, respectively.

\section{CONCLUSION}

In conclusion, we demonstrated that the efficiency of betatron sources can be significantly improved by using longitudinal and transverse density gradients. The radiation produced has a critical energy 10 times higher than the betatron radiation produced in a homogeneous plasma. While the number of photons remains in the range of a few $10^{5}$ photons/pulse $/ 0.1 \% \mathrm{BW}$ at $1 \mathrm{keV}$ with and without tailoring, it is increased by almost 2 orders of magnitude in the hard $\mathrm{x}$-ray region. At $30 \mathrm{keV}$ the source brightness is estimated to be of the order of $10^{5}$ photons/pulse/ $0.1 \% \mathrm{BW}$. We anticipate that this progress will represent a significant milestone in the development of tabletop femtosecond $\mathrm{x}$-ray sources since this method can be applied to all betatron sources and can be combined with the recent developments in laser-plasma accelerators to further improve the source performances [35]. Moreover, it opens a novel route toward the production of a $\mathrm{keV}$ compact betatron source produced with a few terawatt-class lasers with repetition rates up to a kilohertz.

\section{ACKNOWLEDGMENTS}

M. K. and U.C. would like to acknowledge the project ADONIS (CZ 02.1.01/0.0/0.0/16-019/0000789) from ERDF and the Project LQ1606 obtained with the financial support of the Ministry of Education, Youth and Sports as part of targeted support from the National Programme of Sustainability II. This work was supported by Gerry Schwartz and Heather Reisman, Israel Science Foundation, and VATAT supports. This project has received funding from the European Union's Horizon 2020 Research and Innovation programme under Grant Agreement No. 730871(project ARIES).

[1] B. Mahieu, N. Jourdain, K. T. Phuoc, F. Dorchies, J. P. Goddet, A. Lifschitz, P. Renaudin, and L. Lecherbourg, Probing Warm Dense Matter Using Femtosecond X-Ray Absorption Spectroscopy with a Laser-Produced Betatron Source, Nat. Commun. 9, 3276 (2018).

[2] K. Ta Phuoc, R. Fitour, A. Tafzi, T. Garl, N. Artemiev, R. Shah, F. Albert, D. Boschetto, A. Rousse, D.-E. Kim, A. Pukhov, V. Seredov, and I Kostyukov, Demonstration of the Ultrafast Nature of Laser Produced Betatron Radiation, Phys. Plasmas 14, 080701 (2007).
[3] S. Fourmaux, S. Corde, K. T. Phuoc, P. Lassonde, G. Lebrun, S. Payeur, F. Martin, S. Sebban, V. Malka, A. Rousse, and J.C. Kieffer, Single Shot Phase Contrast Imaging Using Laser-Produced Betatron X-Ray Beams, Opt. Lett. 36, 2426 (2011).

[4] J. Wenz, S. Schleede, K. Khrennikov, M. Bech, P. Thibault, M. Heigoldt, F. Pfeiffer, and S. Karsch, Quantitative X-Ray Phase-Contrast Microtomography from a Compact LaserDriven Betatron Source, Nat. Commun. 6, 7568 (2015).

[5] J. M. Cole, J. C. Wood, N. C. Lopes, K. Poder, R. L. Abel, S. Alatabi, and J. S. J. Bryant, Laser-Wakefield Accelerators as Hard X-Ray Sources for 3D Medical Imaging of Human Bone, Sci. Rep. 5, 13244 (2015).

[6] S. Corde, C. Thaury, K. T. Phuoc, A. Lifschitz, G. Lambert, J. Faure, O. Lundh, E. Benveniste, A. Ben-Ismail, L. Arantchuk, A. Marciniak, A. Stordeur, P. Brijesh, A. Rousse, A. Specka, and V. Malka, Mapping the X-Ray Emission Region in a Laser-Plasma Accelerator, Phys. Rev. Lett. 107, 215004 (2011).

[7] M. C. Downer and R. Zgadzaj, Diagnostics for PlasmaBased Electron Accelerators, Rev. Mod. Phys. 90, 035002 (2018).

[8] S. Kiselev, A. Pukhov, and I. Kostyukov, X-Ray Generation in Strongly Nonlinear Plasma Waves, Phys. Rev. Lett. 93, 135004 (2004).

[9] A. Rousse, K. T. Phuoc, R. Shah, A. Pukhov, E. Lefebvre, V. Malka, S. Kiselev, F. Burgy, J. P. Rousseau, D. Umstadter, and D. Hulin, Production of a keV X-Ray Beam from Synchrotron Radiation in Relativistic Laser-Plasma Interaction, Phys. Rev. Lett. 93, 135005 (2004).

[10] S. Corde, K. T. Phuoc, G. Lambert, R. Fitour, V. Malka, A. Rousse, A. Beck, and E. Lefebvre, Femtosecond X Rays from Laser-Plasma Accelerators, Rev. Mod. Phys. 85, 1 (2013).

[11] J. D. Jackson, Classical Electrodynamics (Wiley, New York, 1962).

[12] O. Lundh, J. Lim, C. Rechatin, L. Ammoura, X. Davoine, G. Gallot, J.-P. Goddet, E. Lefebvre, V. Malka, and J. Faure, Few Femtosecond, Few Kiloampere Electron Bunch Produced by a Laser Plasma Accelerator, Nat. Phys. 7, 219 (2011).

[13] K. Ta Phuoc, F. Burgy, J. P. Rousseau, V. Malka, A. Rousse, R. Shah, D. Umstadter, A. Pukhov, and S. Kiselev, Laser Based Synchrotron Radiation, Phys. Plasmas 12, 023101 (2005).

[14] M. Mo, S. Fourmaux, A. Ali, P. Lassonde, J.-C. Kieffer, and R. Fedosejevs, Characterization of Laser Wakefield Generated Betatron X-Ray Radiation Using Grazing Incidence Mirror Reflection, Eur. Phys. J. D 68, 301 (2014).

[15] M. Schnell, A. Sävert, I. Uschmann, O. Jansen, M. C. Kaluza, and C. Spielmann, Characterization and Application of Hard X-Ray Betatron Radiation Generated by Relativistic Electrons from a Laser-Wakefield Accelerator, J. Plasma Phys. 81, 475810401 (2015).

[16] A. Döpp, B. Mahieu, A. Lifschitz, . Thaury, A. Doche, E. Guillaume, G. Grittani, O. Lundh, M. Hansson, J. Gautier, M. Kozlova, J. P. Goddet, P. Rousseau, A. Tafzi, V. Malka, A. Rousse, S. Corde, and K. T. Phuoc, Stable Femtosecond $X$-Rays with Tunable Polarization from a Laser-Driven Accelerator, Light Sci. Appl. 6, e17086 (2017). 
[17] W. P. Leemans, B. Nagler, A. J. Gonsalves, C. Toth, K. Nakamura, C. G. R. Geddes, E. Esarey, C. B. Schroeder, and S. M. Hooker, GeV Electron Beams from a CentimetreScale Accelerator, Nat. Phys. 2, 696 (2006).

[18] H. T. Kim, K. H. Pae, H. J. Cha, I. J. Kim, T. J. Yu, J. H. Sung, S. K. Lee, T. M. Jeong, and J. Lee, Enhancement of Electron Energy to the Multi-GeV Regime by a Dual-Stage Laser-Wakefield Accelerator Pumped by Petawatt Laser Pulses, Phys. Rev. Lett. 111, 165002 (2013).

[19] H. T. Kim, V. B. Pathak, K. H. Pae, A. Lifschitz, F. Sylla, J. H. Shin, C. Hojbota, S. K. Lee, J. H. Sung, H. W. Lee, E. Guillaume, C. Thaury, K. Nakajima, J. Vieira, L. O. Silva, V. Malka, and C. H. Nam, Stable Multi-GeV Electron Accelerator Driven by Waveform-Controlled PW Laser Pulses, Sci. Rep. 7, 10203 (2017).

[20] S. Kneip et al., Bright Spatially Coherent Synchrotron X-Rays from a Table-Top Source, Nat. Phys. 6, 980 (2010).

[21] X. Wang, R. Zgadzaj, N. Fazel, S. A. Yi, X. Zhang, W. Henderson, Y. Y. Chang, R. Korzekwa, H. E. Tsai, C. H. Pai et al., Petawatt-Laser-Driven Wakefield Acceleration of Electrons to $2 \mathrm{GeV}$ in $10^{17} \mathrm{~cm}^{-3}$ Plasma, AIP Conf. Proc. 1507, 341 (2012).

[22] N. Fazel, X. Wang, R. Zgadzaj, Z. Li, X. Zhang, W. Henderson, H. Quevedo, G. Dyer, E. Gaul, M. Martinez, M. Spinks, M. Donovan, V. Khudik, G. Shvets, T. Ditmire, and M.C. Downer, Betatron X-Rays from GeV LaserPlasma-Accelerated Electrons, AIP Conf. Proc. 1777, 080005 (2016).

[23] J. C. Wood, D. J. Chapman, K. Poder, N. C. Lopes, M. E. Rutherford, T. G. White, F. Albert, K. T. Behm, N. Booth, J. S. J. Bryant et al., Ultrafast Imaging of Laser Driven Shock Waves Using Betatron X-Rays from a Laser Wakefield Accelerator, Sci. Rep. 8, 11010 (2018).

[24] W. Lu, C. Huang, M. Zhou, W. B. Mori, and T. Katsouleas, Nonlinear Theory for Relativistic Plasma Wakefields in the Blowout Regime, Phys. Rev. Lett. 96, 165002 (2006).

[25] K. T. Phuoc, E. Esarey, V. Leurent, E. Cormier-Michel, C. G. R. Geddes, C. B. Schroeder, A. Rousse, and W. P. Leemans, Betatron Radiation from Density Tailored Plasmas, Phys. Plasmas 15, 063102 (2008).

[26] C. Yu, J. Liu, W. Wang, W. Li, R. Qi, Z. Zhang, Z. Qin, J. Liu, M. Fang, K. Feng, Y. Wu, L. Ke, Yu. Chen, C. Wang, Y. $\mathrm{Xu}$, Y. Leng, C. Xia, R. Li, and Z. Xu, Enhanced Betatron Radiation by Steering a Laser-Driven Plasma Wakefield with a Tilted Shock Front, Appl. Phys. Lett. 112, 133503 (2018).
[27] W. Rittershofer, C. B. Schroeder, E. Esarey, F. J. Grüner, W. P. Leemans, W. Rittershofer, C. B. Schroeder, E. Esarey, F. J. Grüner, and W. P. Leemans, Tapered Plasma Channels to Phase-Lock Accelerating and Focusing Forces in LaserPlasma Accelerators, Phys. Plasmas 17, 063104 (2010).

[28] E. Guillaume, A. Döpp, C. Thaury, K. T. Phuoc, A. Lifschitz, G. Grittani, J. P. Goddet, A. Tafzi, S. W. Chou, L. Veisz, and V. Malka, Electron Rephasing in a Laser-Wakefield Accelerator, Phys. Rev. Lett. 115, 155002 (2015).

[29] C. Thaury, K. T. Phuoc, S. Corde, P. Brijesh, G. Lambert, S. P. D. Mangles, M. S. Bloom, S. Kneip, and V. Malka, Probing Electron Acceleration and X-Ray Emission in Laser-Plasma Accelerators, Phys. Plasmas 20, 063101 (2013).

[30] C.-H. Pai, S.-Y. Huang, C.-C. Kuo, M.-W. Lin, J. Wang, S.-Y. Chen, C.-H. Lee, and J.-Y. Lin, Fabrication of Spatial Transient-Density Structures as High-Field Plasma Photonic Devices, Phys. Plasmas 12, 070707 (2005).

[31] K. T. Phuoc, F. Burgy, J.-P. Rousseau, V. Malka, A. Rousse, R. Shah, D. Umstadter, A. Pukhov, and S. Kiselev, Laser Based Synchrotron Radiation, Phys. Plasmas 12, 023101 (2005).

[32] H. Jasak, OpenFoam: Open Source CFD in Research and Industry, Int. J. Naval Architect. Ocean Eng. 1, 89 (2009).

[33] K. T. Phuoc, S. Corde, R. Shah, F. Albert, R. Fitour, J. P. Rousseau, F. Burgy, B. Mercier, and A. Rousse, Imaging Electron Trajectories in a Laser-Wakefield Cavity Using Betatron X-Ray Radiation, Phys. Rev. Lett. 97, 225002 (2006).

[34] R. Lehe, M. Kirchen, I. A. Andriyash, B. B. Godfrey, and J.-L. Vay, A Spectral, Quasi-Cylindrical and DispersionFree Particle-in-Cell Algorithm, Comput. Phys. Commun. 203, 66 (2016).

[35] J. P. Couperus, R. Pausch, A. Köhler, O. Zarini, J. M. Krämer, M. Garten, A. Huebl, R. Gebhardt, U. Helbig, S. Bock, K. Zeil, A. Debus, M. Bussmann, U. Schramm, and A. Irman, Demonstration of a Beam Loaded NanocoulombClass Laser Wakefield Accelerator, Nat. Commun. 8, 487 (2017).

Correction: The surname of the seventh author contained an error and has been fixed. 